\title{
Validation of erythropoietin use data on Medicare's End-Stage Renal Disease Medical Evidence Report
}

\author{
Michael J. Fischer, MD, MSPH; ${ }^{1-2^{*}}$ Kevin T. Stroupe, PhD; ${ }^{2-4}$ Denise M. Hynes, RN, MPH, PhD; ${ }^{2,4-5}$ Pierre \\ Blemur, MD; ${ }^{1}$ Min-Woong Sohn; ${ }^{2-3}$ Margaret M. Browning, PhD; ${ }^{2,4}$ Zhiping Huo, MS; $^{2}$ Ann M. O’Hare, MD; ${ }^{6}$ \\ James S. Kaufman, MD $^{7}$ \\ ${ }^{1}$ Medicine/Nephrology, Jesse Brown Department of Veterans Affairs (VA) Medical Center and University of Illinois \\ Medical Center, Chicago, IL; ${ }^{2}$ Center for Management of Complex Chronic Care, Edward Hines Jr. VA Hospital, \\ Hines, IL; ${ }^{3}$ Institute for Healthcare Studies, Northwestern University, Chicago, IL; ${ }^{4}$ VA Information Resource Center, \\ Edward Hines Jr. VA Hospital, Hines, IL; ${ }^{5}$ Medicine/Health Promotion Research, University of Illinois at Chicago, \\ Chicago, IL; ${ }^{6}$ Medicine/Nephrology, VA Puget Sound Healthcare System, Seattle, WA; ${ }^{7}$ Medicine/Nephrology, VA \\ Boston Healthcare System and Boston University, Boston, MA
}

\begin{abstract}
Data from Medicare's End-Stage Renal Disease Medical Evidence Report (Form 2728) suggest that underuse of erythropoiesis-stimulating agents (ESAs) may be contributing to anemia in predialysis patients. However, the data quality of Form 2728 is not known. ESA prescription records were confirmed in Department of Veterans Affairs (VA) data sets and/or ESA claims in Medicare files and compared with data collected on Form 2728 among 8,033 veterans who initiated dialysis in 2000 and 2001 and were eligible for both VA and Medicare coverage in the 12 months preceding dialysis initiation. Among the cohort, predialysis ESA use was found in $4 \%(n=323)$ more veterans by VA/Medicare data sets $(n=2,810)$ than by Form $2728(n=2,487)$. With the use of VA/Medicare data sets (gold standard), the accuracy of Form 2728 for predialysis ESA use was sensitivity $57.0 \%$, specificity $83.1 \%$, positive predictive value $64.5 \%$, negative predictive value $78.2 \%$, and kappa coefficient 0.41 . Sensitivity for reported predialysis ESA use on Form 2728 was lowest among veterans who were female and nonwhite, of low socioeconomic status, and with anemia or other comorbid illnesses. The poor sensitivity and specificity of predialysis ESA use data on Form 2728 raise concerns about the validity of previous reports and study findings. Investigators should recognize these shortcomings and the introduction of possible bias in future research and reports.
\end{abstract}

Key words: accuracy, elderly, end-stage renal disease, erythropoiesis, Medicare, predialysis, sensitivity, specificity, stimulating agent, validity, veteran.

\footnotetext{
Abbreviations: $\mathrm{CI}=$ confidence interval, $\mathrm{CKD}=$ chronic kidney disease, CMS = Centers for Medicare and Medicaid Services, $\mathrm{COPD}=$ chronic obstructive pulmonary disease, $\mathrm{ESA}=$ erythropoiesis-stimulating agent, ESRD = end-stage renal disease, HSR\&D = Health Services Research and Development Service, $\mathrm{NPV}=$ negative predictive value, $\mathrm{PBM}=$ Pharmacy Benefits Management, $\mathrm{PPV}=$ positive predictive value, $\mathrm{PSSG}=$ Planning Systems Support Group, RRT = renal replacement therapy, USRDS = United States Renal Data System, VA = Department of Veterans Affairs.

*Address all correspondence to Michael J. Fischer, MD, MSPH; Center for Management of Complex Chronic Care, Edward Hines Jr. VA Hospital and Jesse Brown VA Medical Center, 5000 South 5th Ave (151H), Hines, IL 60141; 708-202-2414; fax: 708-202-2316. Email: fischerm@uic.edu DOI:10.1682/JRRD.2009.08.0108
} 


\section{INTRODUCTION}

The incidence and prevalence of end-stage renal disease (ESRD) continue to increase substantially in the United States [1-2]. Prior analyses have found that veterans are at high risk for developing chronic kidney disease (CKD), the precursor to ESRD, and rates of morbidity and mortality are extremely high among veterans with kidney disease [3-5]. At onset of ESRD, the Centers for Medicare and Medicaid Services (CMS) require an ESRD Medical Evidence Report (Form 2728) be completed to certify the patient's need for renal replacement therapy and to signal eligibility for Medicare's ESRD entitlement program. Demographic, medical comorbidity, and laboratory data from Form 2728 have been used for both administrative and research purposes. The United States Renal Data System (USRDS), a comprehensive registry of all patients with ESRD in the United States, publishes annual reports describing the characteristics and clinical attributes of the population with ESRD based on information from Form 2728 [1-2]. CMS dialysis networks across the United States also use data from this source to assess quality of predialysis and dialysis care and to target quality improvement initiatives. Furthermore, researchers have used information from Form 2728 in a large number of clinical, epidemiological, and health services research studies [6-10].

However, we are aware of only one prior study that has attempted to validate any of the Form 2728 information. This study found significant underreporting of major comorbid health conditions compared with reporting of these conditions in patient medical records as a gold standard [11]. This finding raises doubts about the reporting and integrity of other data elements on Form 2728, including the use of erythropoiesis-stimulating agents (ESAs) in patients before attaining ESRD and starting renal replacement therapy (predialysis period). ESAs are recommended and used to correct severe anemia, which occurs frequently as a complication of severe CKD, and may ameliorate many of the negative sequelae of anemia, which include lower quality of life and increased hospitalization, cardiovascular complications, and mortality [12-20]. Recent USRDS reports and clinical studies have suggested that ESA underuse in patients with pre-ESRD may be contributing to the unacceptable severity of anemia in these patients at initiation of dialysis [1-2,6$10,15,19]$. To better understand the quality of the data underpinning these observations, we investigated the accuracy and completeness of predialysis ESA use data on Form 2728 by comparing them with Department of Veterans Affairs (VA) pharmacy prescription records and Medicare claims records in a cohort of veterans reliant on VA and/or Medicare-covered services. We also examined whether demographic or clinical factors existed that are associated with the accuracy of the ESA use data.

\section{METHODS}

\section{Study Design and Sample}

We conducted a retrospective analysis of receipt of ESAs among elderly veterans (aged $\geq 66$ years) who initiated chronic dialysis in 2000 and 2001 and were eligible for both VA and Medicare coverage in the 12 months preceding dialysis initiation. We chose this study population because we were able to confirm their receipt of predialysis ESA using prescription records and claims data, independent of information reported on Form 2728. To identify the study cohort, we used the crosswalk file made available to the VA Information Resource Center [21] from the USRDS, which identifies veterans eligible for VA-covered services who have been registered as patients with ESRD [22]. Veterans eligible for VA-covered services were defined as individuals who used VA healthcare services, were enrolled in the Veterans Health Administration, or received a pension or compensation from the VA. The initiation date of dialysis was identified with the USRDS Patients File [22]. We limited the cohort to veterans initiating chronic dialysis between January 1, 2000, and December 31, 2001, and defined the 12-month period preceding dialysis initiation as the predialysis period. ${ }^{*}$

We further restricted our sample to veterans who were 66 years old at dialysis initiation to ensure that veterans were also eligible for Medicare-covered services throughout the predialysis period. To ensure adequate capture of healthcare use information including ESA data, we excluded veterans who were enrolled in Medicare but did not have Medicare as their primary payer during this period, were enrolled in a Medicare health-maintenance organization, or had no healthcare use in either the VA or Medicare during the predialysis period [23].

\footnotetext{
* In this article, we refer to renal replacement therapy (RRT) as dialysis since $99.6 \%$ of the study cohort's RRT was chronic dialysis $(0.4 \%$ underwent kidney transplantation).
} 


\section{ESA Data Collection}

\section{VA Data Sets and Medicare Files}

ESA use was ascertained from VA pharmacy records (Pharmacy Benefits Management [PBM], Fee Basis) and/ or ESA claims under Medicare files (Carrier/Inpatient/ Outpatient/durable medical equipment) during the 12month predialysis period. PBM data capture medications dispensed from VA pharmacies. A dispensation of "erythropoietin" or "darbepoietin" in such data was consistent with receipt of ESA in the VA. Any occurrence of current procedural terminology codes for ESAs in Medicare claims data was deemed consistent with receipt of ESA under Medicare.

\section{Form 2728}

The CMS ESRD Medical Evidence Report, from here on known as Form 2728, is required for all patients reaching ESRD requiring RRT and becoming eligible for Medicare's ESRD program. On the front page of Form 2728 (version 6-97), question 17 states, "Was predialysis/ transplant EPO [erythropoietin] administered?” Possible responses include "yes” or "no." A “yes" response was considered to indicate ESA was administered in the predialysis period, while a "no" response was considered to indicate ESA was not administered in the predialysis period. Nonresponses were considered to indicate that ESA was not administered but were also excluded and examined in a sensitivity analysis.

\section{Variables}

We obtained data on veteran characteristics from VA Inpatient and Outpatient Medical SAS [SAS Institute Inc, Cary, North Carolina] data sets, the Medicare Denominator file, and the USRDS Patients File and Form 2728 [22,24-25]. Comorbidities were assigned on the basis of diagnostic and procedure codes in the inpatient and outpatient VA data sets and Medicare claims data [26]. Anemia was defined as serum hemoglobin $<10 \mathrm{~g} / \mathrm{dL}$, as reported in Form 2728, and supplemented with the VA Decision Support System Laboratory Results file [27]. VA priority level was defined as "high" for veterans with a serviceconnected condition or whose income was less than a VAestablished annual threshold (VA priority groups 1-6), "low" for those whose income was greater than the annual income threshold, and "missing" for veterans with no designation available [28]. To account for socioeconomic status, we linked information about the patients' ZIP Code of residence obtained from the VA Planning Systems Sup- port Group (PSSG) with information from the 2000 Census [29], including ZIP Code-based median household income, education levels, and county unemployment rate from the Area Resource File [30-31]. In addition to including veteran census region, the urban/rural nature of a ZIP Code was obtained from the VA PSSG [29].

We also classified veterans by the healthcare system(s) in which they received outpatient healthcare during the predialysis period: (1) VA outpatient care only (VA-only), (2) VA and Medicare outpatient care (dual), and (3) Medicare outpatient care only (Medicare-only). Adapting a previously developed algorithm [32], we determined the healthcare system from VA and/or Medicare encounters in the following types of outpatient care: primary, specialty, auxiliary clinic, and psychiatric. Any single VA or Medicare encounter for any of these four groups was considered evidence of healthcare use in that particular system. We identified episodes of outpatient nephrology care during the 12-month predialysis period using both VA outpatient administrative data and Medicare carrier files. Nephrology care was defined as the presence of any of the following during the predialysis period: nephrology clinic visit (VA), outpatient hypertension clinic visit with a nephrology provider (VA), and nephrology provider visit (Medicare). Because visits coded as level 1 in Medicare do not require interaction with a nephrology practitioner, these visits were excluded.

\section{Statistical Methods}

Using previously published methods [11,33-34], we categorized veterans according to predialysis ESA use reported on Form 2728 in relation to ESA prescription records in VA data sets and ESA claims in Medicare files during the 12-month predialysis period (Table 1).

Using the number of veterans in each group (Table 1), we computed four comparison statistics using VA data sets and/or Medicare files as the gold standard:

- $\mathrm{A}=$ true positive.

- $\mathrm{B}=$ false positive.

- $\mathrm{C}=$ false negative.

- $\mathrm{D}=$ true negative.

Sensitivity indicates the percentage of subjects with predialysis ESA use that was correctly recorded on Form 2728 and was computed as the ratio of true positives to true positives plus false negatives $(\mathrm{A} /(\mathrm{A}+\mathrm{C}))$. Specificity refers to the percentage of subjects without predialysis ESA use that was correctly recorded on 
Table 1.

Definitions of four groups for assessing accuracy of erythropoiesisstimulating agent (ESA) data in Medicare's End-Stage Renal Disease Medical Evidence Report.

\begin{tabular}{|c|c|}
\hline Group & Definition \\
\hline A. True Positive & $\begin{array}{l}\text { Veterans received predialysis ESAs on } \\
\text { Form } 2728 . \text { A prescription for ESAs } \\
\text { was present in VA/Medicare data sets. }\end{array}$ \\
\hline B. False Positive & $\begin{array}{l}\text { Veterans received predialysis ESAs on } \\
\text { Form } 2728 \text {. A prescription for ESAs } \\
\text { was not present in VA/Medicare data } \\
\text { sets. }\end{array}$ \\
\hline C. False Negative & $\begin{array}{l}\text { Veterans did not receive predialysis } \\
\text { ESAs on Form 2728. A prescription for } \\
\text { ESAs was present in VA/Medicare data } \\
\text { sets. }\end{array}$ \\
\hline D. True Negative & $\begin{array}{l}\text { Veterans did not receive predialysis } \\
\text { ESAs on Form } 2728 \text {. A prescription for } \\
\text { ESAs was not present in VA/Medicare } \\
\text { data sets. }\end{array}$ \\
\hline
\end{tabular}

Form 2728 and computed as the ratio of true negatives to true negatives plus false positives $(\mathrm{D} /(\mathrm{D}+\mathrm{B}))$. Positive predictive value (PPV) refers to the percentage of subjects that was identified as having predialysis ESA use on Form 2728 and actually had such use and was computed as the ratio of true positives to true positives plus false positives $(A /(A+B))$. Negative predictive value (NPV) refers to the percentage of subjects that was identified as not having predialysis ESA use on Form 2728 and did not have such use and was computed as the ratio of true negatives to true negatives plus false negatives $(D /(D+C))$. As a measure of agreement between Form 2728 and VA data sets and/or Medicare files regarding predialysis ESA use data, a $\kappa$ coefficient was calculated as (observed agreement chance agreement $) /(1$ - chance agreement), where observed agreement $=\mathrm{A}+\mathrm{D} /(\mathrm{A}+\mathrm{B}+\mathrm{C}+\mathrm{D})$ and chance agreement $=(\mathrm{C}+\mathrm{D}) /(\mathrm{A}+\mathrm{B}+\mathrm{C}+\mathrm{D}) \times(\mathrm{B}+$ $\mathrm{D}) /(\mathrm{A}+\mathrm{B}+\mathrm{C}+\mathrm{D})+(\mathrm{A}+\mathrm{C}) /(\mathrm{A}+\mathrm{B}+\mathrm{C}+\mathrm{D}) \times(\mathrm{A}+$ $\mathrm{B}) /(\mathrm{A}+\mathrm{B}+\mathrm{C}+\mathrm{D})$.

We computed sensitivity, specificity, PPV, NPV, and $\kappa$ coefficient for the overall cohort, demographic subgroups of the cohort, and outpatient care subgroups of the cohort. All analyses were conducted with the use of STATA SE version 9.03 (StataCorpLP; College Station, Texas).

\section{RESULTS}

\section{Participants}

The final analytic cohort comprised 8,033 veterans after excluding 4,453 veterans: 2,453 who did not receive outpatient care from either the VA or Medicare during the predialysis period, 1,318 who were enrolled in Medicare managed care plans, and 682 who did not have Medicare as their primary payer. Of the veterans, 17 percent were VA-only ( $n=1,395), 44$ percent were dual $(n=3,545)$, and 39 percent were Medicare-only $(n=3,093)$ outpatient care users. Sixty-three percent of veterans received predialysis nephrology care $(n=5,059)$, while thirtyseven percent did not $(n=2,974)$.

\section{Descriptive Data}

More than 80 percent of the cohort were elderly nonHispanic white veterans (Table 2). More than 50 percent of the study veterans had diabetes mellitus, hypertension, or congestive heart failure, while approximately 23 percent or more had peripheral vascular disease, coronary artery disease, or chronic obstructive pulmonary disease (COPD). Approximately 45 percent of the cohort had either Medicaid or no additional health insurance beyond VA and Medicare coverage. Nearly 1 in 5 veterans resided in counties or ZIP Codes with low median household income, a low percentage of college graduates, or high unemployment. Over 80 percent of veterans lived in urban ZIP Codes from all regions of the United States.

\section{Accuracy of ESA Data on Form 2728}

\section{Overall}

Among 8,033 veterans in the final cohort (Table 3), predialysis ESA use was found in 4 percent $(n=323)$ more veterans by VA/Medicare data sets $(n=2,810)$ than by Form $2728(n=2,487)$. The overall accuracy of Form 2728 for predialysis ESA use was low: sensitivity 57.0 percent, specificity 83.1 percent, PPV 64.5 percent, and NPV 78.2 percent. Agreement between the two data sources was also fair with a $\kappa$ coefficient in abstract and of 0.41 . A sensitivity analysis was also performed, excluding those subjects with missing responses for predialysis ESA on Form $2728(n=196)$, and the results were not significantly different. 
Table 2.

Characteristics of veterans $(N=8,033)$.

\begin{tabular}{|c|c|}
\hline Characteristic & Overall \% (n) \\
\hline \multicolumn{2}{|l|}{ Age (yr) } \\
\hline$\geq 66$ to 74 & $38.6(3,104)$ \\
\hline$>74$ & $61.4(4,929)$ \\
\hline \multicolumn{2}{|l|}{ Sex } \\
\hline Male & $97.5(7,831)$ \\
\hline Female & $2.5(202)$ \\
\hline \multicolumn{2}{|l|}{ Race } \\
\hline White & $81.2(6,521)$ \\
\hline African American & $16.4(1,314)$ \\
\hline Other & $2.4(198)$ \\
\hline \multicolumn{2}{|l|}{ Hispanic } \\
\hline Yes & $5.8(470)$ \\
\hline No & $94.2(7,563)$ \\
\hline \multicolumn{2}{|l|}{ Body Mass Index (kg) } \\
\hline$\geq 30$ & $17.0(1,363)$ \\
\hline$<30$ & $83.0(6,670)$ \\
\hline \multicolumn{2}{|l|}{ Comorbidities } \\
\hline Diabetes Mellitus & $55.0(4,417)$ \\
\hline Hypertension & $89.9(7,220)$ \\
\hline Peripheral Vascular Disease & $25.4(2,041)$ \\
\hline Coronary Artery Disease & $23.2(1,862)$ \\
\hline Congestive Heart Failure & $56.6(4,546)$ \\
\hline Stroke & $15.6(1,253)$ \\
\hline COPD & $32.8(2,633)$ \\
\hline Anemia & $44.7(3,594)$ \\
\hline \multicolumn{2}{|l|}{ Additional Insurance } \\
\hline None & $34.3(2,753)$ \\
\hline Medicaid & $10.6(850)$ \\
\hline Private & $55.1(4,430)$ \\
\hline \multicolumn{2}{|l|}{ VA Priority Level } \\
\hline High & $66.0(5,298)$ \\
\hline Low & $20.6(1,656)$ \\
\hline Missing & $13.4(1,079)$ \\
\hline \multicolumn{2}{|c|}{ Median Household Income by ZIP Code (\$U.S.)* } \\
\hline$<30,000$ & $22.2(1,785)$ \\
\hline$\geq 30,000$ & $77.8(6,248)$ \\
\hline \multicolumn{2}{|c|}{ College Graduate by ZIP Code (\%) } \\
\hline$<10$ & $15.9(1,280)$ \\
\hline$\geq 10$ & $84.1(6,753)$ \\
\hline \multicolumn{2}{|c|}{ Unemployment by ZIP Code by County (\%) } \\
\hline$>6$ & $19.0(1,525)$ \\
\hline$\leq 6$ & $81.0(6,508)$ \\
\hline \multicolumn{2}{|l|}{ Type of ZIP Code } \\
\hline Urban & $81.3(6,530)$ \\
\hline Rural & $18.7(1,503)$ \\
\hline \multicolumn{2}{|l|}{ Region } \\
\hline Northeast & $19.7(1,581)$ \\
\hline Midwest & $26.2(2,106)$ \\
\hline South & $38.9(3,128)$ \\
\hline West & $13.5(1,081)$ \\
\hline Territory & $1.7(137)$ \\
\hline
\end{tabular}

\section{By Anemia}

Sensitivity of predialysis ESA use on Form 2728 was lower among veterans with anemia (hemoglobin $<10 \mathrm{~g} / \mathrm{dL}$ ) at onset of ESRD than those without anemia (52.6\% vs $62.1 \%$ ), while smaller differences existed in other comparison statistics (Table 4). Overall agreement by $\kappa$ coefficient was 0.38 for veterans with anemia compared with 0.44 for veterans without anemia.

\section{By Demographic and Clinical Subgroups}

Differences in comparison statistics existed across multiple demographic and clinical subgroups, especially regarding sensitivity (Table 5). Sensitivity of Form 2728 predialysis ESA use was lower in female (51.4\%, 95\% confidence interval [CI]: 39.2-63.6) compared with male veterans (57.2\%, 95\% CI: 55.3-59.1) as well as AfricanAmerican veterans (53.3\%, 95\% CI: 48.7-57.7) compared with their white counterparts $(58.0 \%, 95 \% \mathrm{CI}$ : 55.9-60.0). Several other socioeconomic factors were associated with lower sensitivity of predialysis ESA use on Form 2728, including Medicaid insurance (48.6\%), low median household income (53.4\%), low proportion of college graduates (54.2\%), and high unemployment (53.5\%). Compared with those without comorbidities, veterans with comorbid conditions such as diabetes, vascular disease, and COPD tended to have a lower sensitivity for reported predialysis ESA use on Form 2728.

\section{By Outpatient Care Subgroups}

Compared with veterans who received outpatient predialysis nephrology care, veterans who did not had a lower sensitivity and $\kappa$ coefficient on Form 2728 for reported predialysis ESA use, 48.1 versus 58.9 percent and 0.31 versus 0.40 , respectively (Table 6). Smaller differences existed in sensitivity among VA-only, dual, and Medicare-only outpatient care users.

\section{DISCUSSION}

To our knowledge, this analysis is the first detailed examination of the accuracy of predialysis ESA data reported on Medicare's Form 2728. We found significant misclassification of predialysis ESA data on Form 2728 in veterans incident to ESRD who required dialysis, with an overall sensitivity of 57.0 percent and specificity of 83.1 percent. Inaccuracy of ESA reporting on Form 2728 yielded prevalence estimates of ESA use that were 4 percent lower 
Table 3.

Overall accuracy of erythropoiesis-stimulating agent (ESA) data in Medicare’s End-Stage Renal Disease Medical Evidence Report (Form 2728) of veterans $(N=8,033)$.

\begin{tabular}{|c|c|c|c|c|c|c|c|}
\hline \multirow{2}{*}{$\begin{array}{l}\text { Predialysis ESA in } \\
\text { Form } 2728\end{array}$} & \multicolumn{2}{|c|}{$\begin{array}{c}\text { Predialysis ESA in } \\
\text { VA/Medicare Data Sets }\end{array}$} & $\begin{array}{l}\text { Sensitivity* } \\
\text { (\%) }\end{array}$ & $\begin{array}{c}\text { Specificity }{ }^{\dagger} \\
(\%)\end{array}$ & $\begin{array}{c}\text { PPV } \ddagger \\
(\%)\end{array}$ & $\begin{array}{c}\text { NPV§ } \\
(\%)\end{array}$ & $\kappa$ \\
\hline & Yes & No & \multicolumn{5}{|c|}{ (95\% Confidence Interval) } \\
\hline Yes & 1,603 & 884 & 57.0 & 83.1 & 64.5 & 78.2 & 0.41 \\
\hline $\mathrm{No}^{\pi}$ & 1,207 & 4,339 & $(55.2-58.9)$ & $(82.0-84.1)$ & $(62.5-66.3)$ & $(77.1-79.3)$ & $(0.39-0.43)$ \\
\hline
\end{tabular}

${ }^{*}$ Sensitivity $=$ true positives/(true positives + false negatives) $\times 100$.

${ }^{\dagger}$ Specificity $=$ true negatives/(true negatives + false positives $) \times 100$.

${ }^{\ddagger} \mathrm{PPV}=$ true positives/(true positives + false positives) $\times 100$.

$\S_{\mathrm{NPV}}=$ true negatives/(true negatives + false negatives) $\times 100$.

"196 of 8,033 (2.4\%) were missing predialysis ESA information on Form 2728 and were considered consistent with no predialysis ESA.

$\mathrm{NPV}=$ negative predictive value, $\mathrm{PPV}=$ positive predictive value, $\mathrm{VA}=$ Department of Veterans Affairs.

Table 4.

Accuracy of erythropoiesis-stimulating agent (ESA) data in Medicare’s End-Stage Renal Disease Medical Evidence Report (Form 2728) by presence of anemia.

\begin{tabular}{|c|c|c|c|c|c|c|c|}
\hline \multirow{2}{*}{$\begin{array}{l}\text { Predialysis ESA in } \\
\text { Form } 2728\end{array}$} & \multicolumn{2}{|c|}{$\begin{array}{c}\text { Predialysis ESA in } \\
\text { VA/Medicare Data Sets }\end{array}$} & $\begin{array}{l}\text { Sensitivity* } \\
(\%)\end{array}$ & $\begin{array}{l}\text { Specificity }{ }^{\dagger} \\
\text { (\%) }\end{array}$ & $\begin{array}{c}\text { PPV } \ddagger \\
(\%)\end{array}$ & $\begin{array}{l}\text { NPV§ } \\
(\%)\end{array}$ & $\kappa$ \\
\hline & \multirow[t]{2}{*}{ Yes } & \multirow[t]{2}{*}{ No } & \multicolumn{5}{|c|}{ (95\% Confidence Interval) } \\
\hline \multicolumn{6}{|l|}{ No Anemia Present } & & \\
\hline Yes & 914 & 467 & 62.1 & 81.3 & 66.2 & 78.5 & 0.44 \\
\hline No & 558 & 2,033 & (59.6-64.6) & (79.7-82.8) & (63.6-68.7) & $(76.8-80.0)$ & $(0.41-0.47)$ \\
\hline \multicolumn{8}{|l|}{ Anemia Present } \\
\hline Yes & 646 & 381 & 52.6 & 83.9 & 62.9 & 77.3 & 0.38 \\
\hline No & 583 & 1,984 & $(49.7-55.4)$ & (82.3-85.4) & (59.9-65.9) & (75.6-78.9) & $(0.35-0.41)$ \\
\hline \multicolumn{8}{|l|}{ Anemia Not Assessed } \\
\hline Yes & 43 & 36 & 39.4 & 89.9 & 54.4 & 83 & 0.32 \\
\hline No & 66 & 322 & (30.2-49.3) & (86.4-92.9) & $(42.8-65.7)$ & (78.9-86.6) & $(0.22-0.43)$ \\
\hline 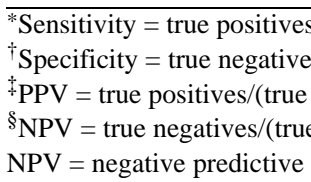 & tives + & $\begin{array}{l}\text { gatives) } \\
\text { ositives) } \\
\text { s) } \times 100 \text {. } \\
\text { ves }) \times 10\end{array}$ & & . & & & \\
\hline
\end{tabular}

than those based on documented ESA use in the VA and/or Medicare. Sensitivity was poor (ranging from $48.6 \%$ to $65.3 \%$ ), and specificity (ranging from $77.1 \%$ to $86.3 \%$ ) was moderate for all groups. Sensitivity was worse in veterans who were female, nonwhite, those of low socioeconomic status, and those with a greater burden of comorbid illness. Only one prior study commented on predialysis ESA data on Form 2728 [35]. In a retrospective cohort analysis of elderly (aged $>66$ years) Medicare recipients incident to hemodialysis from 1995 to 1997, Xue et al. briefly noted that although nearly 24 percent of study participants were reported to receive an ESA before starting dialysis on Form 2728, only 15.6 percent received an ESA before ESRD according to their review of Medicare claims data [35]. Although Medicare was the primary payer for subjects in this study, the authors acknowledged that other payers could be covering ESAs for these Medicare patients and hence explain the higher ESA use reported by Form 2728 versus that found in Medicare claims; however, comparative statistics were not reported. In contrast, our study population was required not only to be Medicare-eligible and to have Medicare as a primary payer but also to be eligible for VA-covered services, thereby diminishing the risk that ESA could have been provided outside of Medicare or VA. Furthermore, we did not find the accuracy of Form 2728 to be worse in veterans who had additional private insurance versus those without additional insurance.

In their evaluation of the accuracy of comorbid conditions on Form 2728, Longenecker et al. noted an overall sensitivity of 59 percent and specificity of 91 percent, 
Table 5.

Accuracy of erythropoiesis-stimulating agent (ESA) data in Medicare’s End-Stage Renal Disease Medical Evidence Report (Form 2728) by demographic and clinical subgroups.

\begin{tabular}{|c|c|c|c|c|c|}
\hline \multirow{2}{*}{ Characteristic } & Sensitivity (\%)* & Specificity (\%) ${ }^{\dagger}$ & PPV (\%) & NPV(\% $(\%$ & $\kappa$ \\
\hline & \multicolumn{5}{|c|}{ (95\% Confidence Interval) } \\
\hline \multicolumn{6}{|l|}{ Age (yr) } \\
\hline$\geq 66$ to 74 & $58.0(55.0-61.0)$ & $83.3(81.5-84.9)$ & $65.1(62.0-68.1)$ & $78.7(76.9-80.4)$ & $0.42(0.39-0.46)$ \\
\hline$>74$ & $56.4(54.1-58.8)$ & $83.0(81.6-84.3)$ & $64.1(61.6-66.5)$ & $78.0(76.5-79.4)$ & $0.40(0.38-0.43)$ \\
\hline \multicolumn{6}{|l|}{ Sex } \\
\hline Male & $57.2(55.3-59.1)$ & $83.2(82.1-84.2)$ & $64.6(62.7-66.6)$ & 78.3 (77.2-79.4) & $0.41(0.39-0.44)$ \\
\hline Female & $51.4(39.2-63.6)$ & $79.5(71.7-86.1)$ & $57.1(44.0-69.5)$ & $75.5(67.5-82.4)$ & $0.32(0.18-0.45)$ \\
\hline \multicolumn{6}{|l|}{ Race } \\
\hline White & $58.0(55.9-60.0)$ & $83.3(82.2-84.4)$ & $64.8(62.7-66.9)$ & $78.9(77.7-80.1)$ & $0.42(0.40-0.45)$ \\
\hline African American & $53.3(48.7-57.7)$ & 82.8 (80.1-85.4) & $65.0(60.1-69.7)$ & 74.8 (71.8-77.5) & $0.37(0.32-0.43)$ \\
\hline Other & $53.4(39.9-66.7)$ & $77.1(69.3-83.8)$ & $49.2(36.4-62.1)$ & $80.0(72.3-86.4)$ & $0.30(0.16-0.44)$ \\
\hline \multicolumn{6}{|l|}{ Hispanic } \\
\hline Yes & $59.5(51.4-67.2)$ & 83.7 (79.1-87.6) & $64.8(56.5-72.6)$ & $80.3(75.6-84.5)$ & $0.44(0.35-0.53)$ \\
\hline No & $56.9(55.0-58.8)$ & $83.0(82.0-84.1)$ & $64.4(62.5-66.4)$ & $78.1(77.0-79.2)$ & $0.41(0.39-0.43)$ \\
\hline \multicolumn{6}{|l|}{ BMI (kg) } \\
\hline$\geq 30$ & $58.8(54.2-63.4)$ & $86.2(83.8-88.3)$ & $67.9(63.0-72.5)$ & $80.8(78.2-83.3)$ & $0.47(0.41-0.52)$ \\
\hline$<30$ & $56.7(54.7-58.7)$ & $82.4(81.3-83.5)$ & $63.8(61.7-65.9)$ & $77.7(76.4-78.9)$ & $0.40(0.38-0.42)$ \\
\hline \multicolumn{6}{|l|}{ Comorbidities } \\
\hline \multicolumn{6}{|l|}{ Diabetes Mellitus } \\
\hline Yes & $55.2(52.8-57.7)$ & 83.0 (81.5-84.4) & $65.6(63.0-68.1)$ & $76.0(74.4-77.5)$ & $0.39(0.37-0.42)$ \\
\hline No & $59.6(56.7-62.4)$ & $83.2(81.6-84.7)$ & $63.1(60.2-65.9)$ & $81.0(79.4-82.5)$ & $0.43(0.40-0.46)$ \\
\hline \multicolumn{6}{|l|}{ Hypertension } \\
\hline Yes & $57.0(55.1-58.9)$ & $82.7(81.6-83.8)$ & $65.6(63.6-67.5)$ & $76.9(75.7-78.1)$ & $0.41(0.39-0.43)$ \\
\hline No & $57.5(49.6-65.1)$ & $85.8(82.8-88.4)$ & $51.1(43.7-58.4)$ & $88.6(85.9-91.0)$ & $0.41(0.34-0.49)$ \\
\hline \multicolumn{6}{|c|}{ Peripheral Vascular Disease } \\
\hline Yes & $54.9(51.1-58.8)$ & $84.0(82.0-85.9)$ & $62.0(57.9-66.0)$ & $79.7(77.6-81.7)$ & $0.40(0.36-0.44)$ \\
\hline No & $57.7(55.6-59.8)$ & $82.7(81.5-83.9)$ & $65.2(63.0-67.3)$ & $77.7(76.4-79.0)$ & $0.41(0.39-0.44)$ \\
\hline \multicolumn{6}{|c|}{ Coronary Artery Disease } \\
\hline Yes & $52.6(48.4-56.7)$ & $85.6(83.5-87.4)$ & $61.4(56.9-65.8)$ & $80.5(78.3-82.5)$ & $0.40(0.35-0.44)$ \\
\hline No & $58.2(56.1-60.2)$ & $82.3(81.0-83.4)$ & 65.2 (63.1-67.3) & 77.5 (76.2-78.8) & $0.41(0.39-0.44)$ \\
\hline \multicolumn{6}{|c|}{ Congestive Heart Failure } \\
\hline Yes & $52.6(50.0-55.1)$ & $83.6(82.2-84.9)$ & $60.9(58.2-63.6)$ & $78.4(76.9-79.8)$ & $0.37(0.35-0.40)$ \\
\hline No & $62.1(59.4-64.7)$ & $82.4(80.7-84.0)$ & $68.3(65.6-70.9)$ & $78.1(76.3-79.7)$ & $0.45(0.42-0.48)$ \\
\hline \multicolumn{6}{|l|}{ Stroke } \\
\hline Yes & $53.9(48.8-59.0)$ & $84.2(81.6-86.6)$ & $59.8(54.4-65.0)$ & 80.8 (78.1-83.3) & $0.39(0.34-0.45)$ \\
\hline No & $57.5(55.5-59.5)$ & $82.9(81.7-84.0)$ & $65.2(63.1-67.2)$ & $77.7(76.5-78.9)$ & $0.41(0.39-0.44)$ \\
\hline \multicolumn{6}{|l|}{ COPD } \\
\hline Yes & $50.9(47.4-54.5)$ & $84.6(82.9-86.2)$ & $58.9(55.1-62.6)$ & 79.9 (78.1-81.7) & $0.37(0.33-0.41)$ \\
\hline No & $59.5(57.3-61.6)$ & $82.3(80.9-83.5)$ & $66.6(64.4-68.8)$ & $77.3(75.9-78.7)$ & $0.43(0.40-0.45)$ \\
\hline \multicolumn{6}{|c|}{ Additional Insurance } \\
\hline None & 56.7 (53.6-59.8) & $86.3(84.6-87.9)$ & $70.3(67.0-73.4)$ & 77.7 (75.8-79.6) & $0.45(0.41-0.48)$ \\
\hline Medicaid & $48.6(42.1-55.0)$ & $83.2(80.0-86.1)$ & $53.6(46.8-60.4)$ & $80.2(76.8-83.2)$ & $0.33(0.26-0.40)$ \\
\hline Private & $58.6(56.1-61.0)$ & $81.1(79.6-82.5)$ & $62.9(60.3-65.3)$ & $78.2(76.6-79.6)$ & $0.40(0.37-0.43)$ \\
\hline \multicolumn{6}{|l|}{ VA Priority Level } \\
\hline High & $56.2(53.9-58.4)$ & $84.0(82.7-85.2)$ & $65.6(63.2-67.9)$ & 77.9 (76.6-79.3) & $0.41(0.39-0.44)$ \\
\hline Low & $62.3(58.4-66.1)$ & $81.4(78.8-83.7)$ & $66.7(62.7-70.6)$ & $78.3(75.7-80.7)$ & $0.44(0.40-0.49)$ \\
\hline Missing & $52.0(46.4-57.5)$ & $81.2(78.2-83.9)$ & $54.3(48.6-60.0)$ & 79.7 (76.7-82.5) & $0.34(0.27-0.40)$ \\
\hline
\end{tabular}


Table 5. (Continued)

Accuracy of erythropoiesis-stimulating agent (ESA) data in Medicare’s End-Stage Renal Disease Medical Evidence Report (Form 2728) by demographic and clinical subgroups.

\begin{tabular}{|c|c|c|c|c|c|}
\hline \multirow{2}{*}{ Characteristic } & Sensitivity (\%)* & Specificity (\%) ${ }^{\dagger}$ & PPV (\%) & NPV(\%)§ & $\kappa$ \\
\hline & \multicolumn{5}{|c|}{ (95\% Confidence Interval) } \\
\hline \multicolumn{6}{|c|}{$\begin{array}{l}\text { Median Household Income by ZIP Code (\$U.S.) } \\
\end{array}$} \\
\hline$<30,000$ & $53.4(49.1-57.7)$ & $84.1(82.0-86.1)$ & $59.5(55.0-63.9)$ & $80.5(78.2-82.6)$ & $0.39(0.34-0.43)$ \\
\hline$\geq 30,000$ & $57.9(55.9-60.0)$ & $82.7(81.5-83.9)$ & $65.6(63.5-67.7)$ & $77.5(76.3-78.8)$ & $0.42(0.39-0.44)$ \\
\hline \multicolumn{6}{|c|}{ College Graduate by ZIP Code (\%) } \\
\hline$<10$ & $54.2(49.1-59.2)$ & $82.3(79.6-84.7)$ & $57.7(52.5-62.8)$ & $80.1(77.3-82.6)$ & $0.37(0.32-0.43)$ \\
\hline \multicolumn{6}{|c|}{ Unemployment by ZIP Code by County (\%) } \\
\hline$>6$ & $53.5(48.9-58.1)$ & $84.8(82.5-87.0)$ & $61.1(56.2-65.8)$ & 80.4 (78.0-82.7) & $0.40(0.35-0.45)$ \\
\hline$\leq 6$ & 57.8 (55.7-59.8) & 82.6 (81.4-83.8) & $65.1(63.0-67.2)$ & 77.7 (76.4-78.9) & $0.41(0.39-0.44)$ \\
\hline \multicolumn{6}{|l|}{ Type of ZIP Code } \\
\hline Urban & $56.6(54.6-58.6)$ & 83.1 (81.9-84.2) & $65.3(63.1-67.3)$ & 77.3 (76.0-78.5) & $0.41(0.38-0.43)$ \\
\hline South & $55.2(52.2-58.2)$ & 85.1 (83.4-86.6) & 66.9 (63.7-69.9) & 77.7 (75.9-79.4) & $0.42(0.39-0.45)$ \\
\hline West & $57.9(52.8-62.9)$ & $81.3(78.2-84.1)$ & $62.7(57.4-67.8)$ & $78.1(74.9-81.0)$ & $0.40(0.34-0.46)$ \\
\hline Territory & $65.3(50.4-78.3)$ & 85.2 (76.1-91.9) & $71.1(55.7-83.6)$ & 81.5 (72.1-88.9) & $0.51(0.36-0.67)$ \\
\hline \multicolumn{6}{|c|}{ 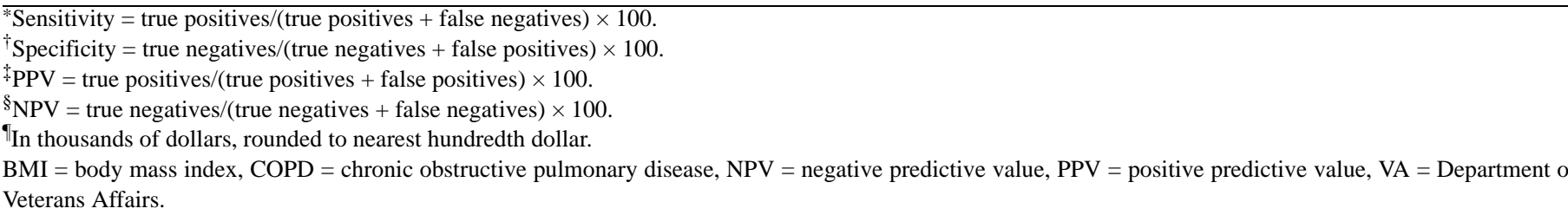 } \\
\hline
\end{tabular}

which were consistent with the degree of under- and overreporting of predialysis ESA in our study [11]. They also noted worse sensitivity in females, African Americans, and those with multiple comorbid conditions. Noteworthy is that the presence of comorbid conditions and use of predialysis ESA on Form 2728 simply require the respondent to mark an appropriate box. The respondent receives no specific training for completing Form 2728. Moreover, tremendous heterogeneity exists in the identity of the respondent because it may be any one or a combination of individuals, including the patient, social worker, nurse, administrator, or nephrologist. In this setting, interpretation and understanding of questions such as "Was predialysis/transplant EPO administered?” and the gathering of patient information to answer such questions in an informed manner are highly variable. In vulnerable populations, such as those characterized by complex comorbid conditions, low socioeconomic status, minority race, and absent predialysis nephrology care, accurate completion of Form 2728 is likely further compromised by issues of patient health literacy, patient education and awareness about CKD and its treatment, and provider-patient communication.

Similar to the implications cited by Longenecker et al., two types of biases may result from the use of Form 2728 predialysis ESA data for administrative and research purposes: (1) underreporting bias of predialysis ESA and (2) differential bias regarding the association of certain characteristics with predialysis ESA [11]. Multiple USRDS reports using Form 2728 data have concluded that ongoing levels of suboptimal degrees of anemia in incident patients with ESRD are in part because of underuse of ESA [17]. These reports have consistently cited lower hemoglobin values in patients without ESA use compared with those with ESA use and have suggested that more appropriate use of predialysis ESA is an important target of quality improvement in patient care. Moreover, recent large observational studies and clinical 
Table 6.

Accuracy of erythropoiesis-stimulating agent (ESA) data in Medicare’s End-Stage Renal Disease Medical Evidence Report (Form 2728) by outpatient care subgroups.

\begin{tabular}{|c|c|c|c|c|c|}
\hline \multirow{2}{*}{$\begin{array}{c}\text { Type of Outpatient } \\
\text { Predialysis Care }\end{array}$} & Sensitivity (\%)* & Specificity (\%) ${ }^{\dagger}$ & PPV (\%) & NPV (\%)§ & $\kappa$ \\
\hline & \multicolumn{5}{|c|}{ (95\% Confidence Interval) } \\
\hline \multicolumn{6}{|c|}{ Predialysis Nephrology Care } \\
\hline Yes & $58.9(56.9-60.9)$ & 80.8 (79.3-82.3) & $72.3(70.3-74.4)$ & $69.8(68.1-71.4)$ & $0.40(0.38-0.43)$ \\
\hline No & $48.1(43.6-52.7)$ & $85.6(84.1-86.9)$ & $39.2(35.2-43.3)$ & 89.5 (88.2-90.7) & $0.31(0.27-0.35)$ \\
\hline \multicolumn{6}{|l|}{ Outpatient Care } \\
\hline VA-Only & $58.4(54.1-62.6)$ & 86.5 (84.0-88.7) & $73.4(68.9-77.5)$ & 76.5 (73.7-79.2) & $0.47(0.42-0.51)$ \\
\hline Medicare-Only & $58.4(55.2-61.6)$ & 80.9 (79.1-82.5) & $56.8(53.6-59.9)$ & $81.9(80.2-83.5)$ & $0.39(0.35-0.42)$ \\
\hline \multicolumn{6}{|c|}{ 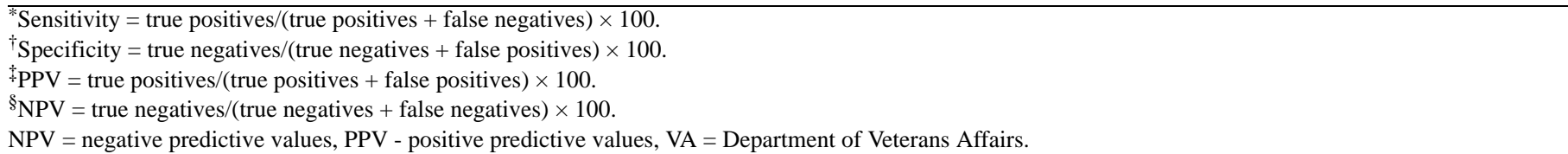 } \\
\hline
\end{tabular}

review articles, which relied on Form 2728 data, have also found that less than one-third of patients with incident dialysis received predialysis ESA and that those with lower hemoglobin values were even less likely to have received predialysis ESA [6-10,15-16,19]. Only one group of investigators expressed concern about predialysis ESA data misclassification on Form 2728, but doubted it was significant because an inverse correlation was found between ESA use and low hemoglobin $(r=-0.65$, $p=0.004)[6,8]$. Our detailed analysis of Form 2728 predialysis ESA data suggests otherwise. While our findings do not entirely discount the observed relationship between lack of ESA use and worse anemia, they do suggest that it has been exaggerated. Especially considering that sensitivity and underreporting were observed as worse in those with anemia compared with those without anemia, these results require a reexamination of this matter and greater attention to other important factors affecting anemia management in patients with pre-ESRD, such as dosing of ESA and iron therapy.

Differential bias is also a potential consequence of using predialysis ESA data on Form 2728. USRDS annual reports have found that African Americans had worse anemia parameters at initiation of dialysis and were less likely to receive ESA than whites [1-2]. Based on Form 2728 data, several epidemiological studies over the past decade have contended that the African-American race, unemployment, and Medicaid insurance are independently associated with a lower likelihood of predialysis ESA [610,36]. While these disparities may exist and be influenced by economic and healthcare access as these investigators contend, our findings suggest that some of these associations may be the result of bias because of poor sensitivity and misclassification of data on Form 2728 in these groups. These potentially erroneous relationships could obfuscate the determination of appropriate subgroups for targeting improvements in pre-ESRD anemia care.

In 2005, a new Form 2728 was adopted and disseminated that contains new information regarding prior ESA therapy in patients with incident ESRD. The question has been restated as "Prior to ESRD therapy, did patient receive exogenous erythropoietin or equivalent?" and the options for responses include "yes," "no," or "unknown.” If "yes" is indicated, then the respondent is further asked if such therapy was given for "6 to 12 months" or ">12 months." This new language and "unknown" response option may enhance the accuracy of Form 2728 ESA data by reducing misunderstanding of the question and allowing for an appropriate choice if the receipt of predialysis ESA is uncertain. Furthermore, the additional specific data requested may improve characterization of predialysis ESA use across the United States. Evaluation of the data quality of this revised Form 2728 is needed to inform administrators and investigators how best to use this information to assess quality of pre-ESRD care and epidemiological research endeavors. In the interim, while the use of predialysis ESA data on Form 2728 offers many advantages, including being inexpensive to use, being readily available, and characterizing a large national patient sample, the data's use is compromised by data misclassification and poor sensitivity. These shortcomings 
and biases should be kept in mind when results are reported and interpreted based on these data.

This study has limitations. First, this study was conducted in a predominantly older male population. Therefore, findings for the accuracy of predialysis ESA data on Form 2728 may not be generalizable to other demographic groups. Second, despite the careful efforts to restrict the cohort to veterans reliant only on the VA and Medicare, predialysis ESA may possibly have been obtained outside of the VA or Medicare, especially in veterans with private insurance or Medicaid. This phenomenon would lower specificity in these analyses because it would inflate the degree of false positives. However, sensitivity was lower than specificity in our analysis and the latter did not vary substantially across key subgroups. Also, PBM data during this time period only captured outpatient ESA use; therefore, ESA use during a VA hospitalization may not be captured by our analysis. In addition, we underscore the importance that ESA use for anemia due to CKD is a chronic therapy that would be unlikely to occur solely during an inpatient period; therefore, this underestimating of VA ESA use is likely not significant. Furthermore, we did not find substantial differences in sensitivity and specificity among veterans whose ESA use was confirmed exclusively in PBM records (i.e., VA-only users) versus exclusively in Medicare claims data (i.e., Medicare-only users). Hence, our findings appear quite robust.

\section{CONCLUSIONS}

Reporting of predialysis ESA use on Form 2728 suffers significant errors in underreporting and overreporting. Investigators should recognize these shortcomings and the introduction of possible bias in prior and future research and national reports. Nonetheless, administrative data remain a readily available and important repository of information for medical research. As has been suggested in the past [37], efforts and coordination among clinicians, researchers, and policy makers are greatly needed to further develop linked electronic medical records and databases to improve the quality of administrative data sources such as Form 2728.

\section{ACKNOWLEDGMENTS}

\section{Additional Contributions:}

Study concept and design: M. J. Fischer, K. T. Stroupe, D. M. Hynes, J. S. Kaufman, P. Blemur, A. M. O’Hare.

Acquisition of data: K. T. Stroupe, Z. Huo, D. M. Hynes, M. M. Browning.

Analysis and interpretation of data: K. T. Stroupe, M. J. Fischer, D. M. Hynes, J. S. Kaufman, A. M. O’Hare, P. Blemur, M-W. Sohn. Drafting of manuscript: M. J. Fischer, K. T. Stroupe. Critical revision of manuscript for important intellectual content: K. T. Stroupe, M. J. Fischer, D. M. Hynes, J. S. Kaufman, A. M. O’Hare, P. Blemur, M-W. Sohn.

Statistical analysis: M. J. Fischer, K. T. Stroupe, Z. Huo. Obtained funding: K. T. Stroupe, D. M. Hynes.

Administrative, technical, or material support: M. M. Browning. Study supervision: K. T. Stroupe, M. J. Fischer, D. M. Hynes.

Financial Disclosures: The authors have declared that no competing interests exist.

Funding/Support: This material was based on work supported by the VA, Veterans Health Administration, Health Services Research and Development Service (HSR\&D) grants IIR 02-244 and IIR 20-016 (awarded to K. Stroupe, D. Hynes, and M. Browning); VA HSR\&D Research Career Scientist Award to D. M. Hynes; VA HSR\&D Career Development Award to M. Fischer; the National Kidney Foundation of Illinois (awarded to M. Fischer); and National Institutes of Health grant K23AG28980 (awarded to A. O’Hare).

Additional Contributions: The views expressed in this article are those of the authors and do not necessarily represent the views of the VA or HSR\&D.

Institutional Review: We obtained data for the research portion of this article after institutional review board approval.

\section{REFERENCES}

1. U.S. Renal Data System: USRDS 2008 Annual Data Report. Bethesda (MD): National Institutes of Health, National Institute of Diabetes and Digestive and Kidney Diseases; 2008.

2. U.S. Renal Data System: USRDS 2005 Annual Data Report. Bethesda (MD): National Institutes of Health, National Institute of Diabetes and Digestive and Kidney Diseases; 2005.

3. Patel UD, Young EW, Ojo AO, Hayward RA. CKD progression and mortality among older patients with diabetes. Am J Kidney Dis. 2005;46(3):406-14. [PMID: 16129201] DOI:10.1053/j.ajkd.2005.05.027

4. O’Hare AM, Bertenthal D, Covinsky KE, Landefeld CS, Sen S, Mehta K, Steinman MA, Borzecki A, Walter LC. Mortality risk stratification in chronic kidney disease: One size for all ages? J Am Soc Nephrol. 2006;17(3):846-53. [PMID: 16452492]

DOI:10.1681/ASN.2005090986 
5. Young BA, Pugh JA, Maynard C, Reiber G. Diabetes and renal disease in veterans. Diabetes Care. 2004;27(Suppl 2): B45-49. [PMID: 15113782]

DOI:10.2337/diacare.27.suppl_2.B45

6. Obrador GT, Ruthazer R, Arora P, Kausz AT, Pereira BJ. Prevalence of and factors associated with suboptimal care before initiation of dialysis in the United States. J Am Soc Nephrol. 1999;10(8):1793-1800. [PMID: 10446948$]$

7. Fink J, Blahut S, Reddy M, Light P. Use of erythropoietin before the initiation of dialysis and its impact on mortality. Am J Kidney Dis. 2001;37(2):348-55. [PMID: 11157377] DOI:10.1053/ajkd.2001.21305

8. Obrador GT, Roberts T, St Peter WL, Frazier E, Pereira BJ, Collins AJ. Trends in anemia at initiation of dialysis in the United States. Kidney Int. 2001;60(5):1875-84.

[PMID: 11703606]

DOI:10.1046/j.1523-1755.2001.00002.x

9. Lu WX, Jones-Burton C, Zhan M, Salzberg DJ, Moore J Jr, Fink JC. Survival benefit of recombinant human erythropoietin administration prior to onset of end-stage renal disease: Variations across surrogates for quality of care and time. Nephron Clin Pract. 2005;101(2):c79-86.

[PMID: 15976508]

DOI:10.1159/000086226

10. Ward MW. Laboratory abnormalities at the onset of treatment of end-stage renal disease: Are there racial or socioeconomic disparities in care? Arch Intern Med. 2007; 167(10):1083-91. [PMID: 17533212]

DOI:10.1001/archinte.167.10.1083

11. Longenecker JC, Coresh J, Klag MJ, Levey AS, Martin AA, Fink NE, Powe NR. Validation of comorbid conditions on the end-stage renal disease medical evidence report: The CHOICE study. Choices for healthy outcomes in caring for ESRD. J Am Soc Nephrol. 2000;11(3):520-29.

[PMID: 10703676]

12. Weisbord SD, Kimmel PL. Health-related quality of life in the era of erythropoietin. Hemodial Int. 2008;12(1):6-15. [PMID: 18271834] DOI:10.1111/j.1542-4758.2008.00233.x

13. Gillespie BS, Inrig JK, Szczech LA. Anemia management in chronic kidney disease. Hemodial Int. 2007;11(1):15-20. [PMID: 17257350] DOI:10.1111/j.1542-4758.2007.00147.x

14. NKF-KOQI clinical practice guidelines for the treatment of anemia of chronic renal failure. National Kidney Foundation-Dialysis Outcomes Quality Initiative. Am J Kidney Dis. 1997;30(Suppl 3):S192-240. [PMID: 9339151]

15. Kausz AT, Obrador GT, Pereira BJ. Anemia management in patients with chronic renal insufficiency. Am. J Kidney Dis. 2000;36(6 Suppl 3):S39-51. [PMID: 11118157] DOI:10.1053/ajkd.2000.19930
16. Rao M, Pereira BJ. Optimal anemia management reduces cardiovascular morbidity, mortality, and costs in chronic kidney disease. Kidney Int. 2005;68(4):1432-38.

[PMID: 16164618]

DOI:10.1111/j.1523-1755.2005.00554.X

17. Wish JB, Coyne DW. Use of erythropoiesis-stimulating agents in patients with anemia of chronic kidney disease: Overcoming the pharmacological and pharmacoeconomic limitations of existing therapies. Mayo Clin Proc. 2007; 82(11):1371-80. [PMID: 17976358]

DOI:10.4065/82.11.1371

18. Collins AJ. Anemia management prior to dialysis: Cardiovascular and cost-benefit observations. Nephrol Dial Transplant. 2003;18(2):ii2-ii6. [PMID: 12819293]

19. McClellan WM, Jurkovitz C, Abramson J. The epidemiology and control of anemia among pre-ESRD patients with chronic kidney disease. Eur J Clin Invest. 2005;35(Suppl 3):58-65. [PMID: 16281960] DOI:10.1111/j.1365-2362.2005.01532.x

20. Cody J, Daly C, Campbell M, Donaldson C, Khan I, Rabindranath K, Vale L, Wallace S, Macleod A. Recombinant human erythropoietin for chronic renal failure anaemia in pre-dialysis patients. Cochrane Database Syst Rev. 2005; 20(3):CD003266. [PMID: 16034896]

21. VA Information Resource Center (VIReC) [Internet]. Washington (DC): Department of Veterans Affairs; 2010. [updated 13 Aug 2010; cited 2009 May 6]. Available from: http://www.virec.research.va.gov/.

22. United States renal data system [Internet]. Minneapolis (MN): USRDS Coordinating Center; 2009 [cited 2009 May 6]. Available from: http://www.usrds.org/.

23. Kausz AT, Guo H, Pereira BJ, Collins AJ, Gilbertson DT. General medical care among patients with chronic kidney disease: Opportunities for improving outcomes. J Am Soc Nephrol. 2005;16(10):3092-3101. [PMID: 16079268] DOI:10.1681/ASN.2004110910

24. VIReC research user guide: FY2006 VHA medical SAS inpatient datasets [Internet]. Hines (IL): VA Information Resource Center; 2007 [cited 2009 May 6]. Available from: http://www.virec.research.va.gov/References/RUG/RUGInpatient06.pdf/.

25. VIReC research user guide: FY2006 VHA medical SAS outpatient datasets [Internet]. Hines (IL): VA Information Resource Center; 2007 [cited 2009 May 6]. Available from: http://www.virec.research.va.gov/References/RUG/RUGOutpatient06er.pdf/.

26. SEER-Medicare: Calculation of comorbidity weights [Internet]. Bethesda (MD): National Cancer Institute; 2009 [updated 2009 Sep 9; cited 2009 May 6]. Available from: http://healthservices.cancer.gov/seermedicare/program/ comorbidity.html/. 
27. VIReC research user guide: VHA Decision Support System (DSS) clinical national data extracts (NDEs) FY2000FY2004 [Internet]. Hines (IL): VA Information Resource Center; 2004 [updated 2005 Aug; cited 2009 May 6]. Available from: http://www.virec.research.va.gov/References/RUG/RUG-DSS00-04.pdf/.

28. Subcommittee on Health, Committee on Veterans' Affairs, House of Representatives. Progress and challenges in providing care to veterans. Statement of Stephen P. Backhus, Director, Veterans' Affairs and Military Health Care Issues, Health, Education, and Human Services Division. 1999 Jul 15. Text from: United States General Accounting Office. Available from: http://www.gao.gov/archive/1999/he99158t.pdf/.

29. Department of Veterans Affairs, Veterans Health Administration, Office of the Under Secretary for Health for Policy and Planning, Planning System Support Group. FY2003 Geographic Access to Veterans Health Administration Services. Gainesville (FL): Department of Veterans Affairs; 2005.

30. Area Resource File, 2002 [Internet]. Health Resources and Services Administration; 2002 [cited 2009 May 6]. Available from: http://arf.hrsa.gov/.

31. ZIP Code tabulation areas (ZCTAs) [Internet]. Washington (DC): U.S. Census Bureau; 2001 [cited 2009 May 6]. Available from: http://www.census.gov/geo/ZCTA/zcta.html/.

32. Weeks WB, Mahar PJ, Wright SM. Utilization of VA and Medicare services by Medicare-eligible veterans: The impact of additional access points in a rural setting. J Health Manag. 2005;50(2):95-106. [PMID: 15839324]

33. Van Stralen KJ, Stel VS, Reitsma JB, Dekker FW, Zoccali C, Jager KJ. Diagnostic methods I: Sensitivity, specificity, and other measures of accuracy. Kidney Int. 2009;75(12): 1257-63. [PMID: 19340091] DOI:10.1038/ki.2009.92
34. Sohn MW, Arnold N, Maynard C, Hynes DM. Accuracy and completeness of mortality data in the Department of Veterans Affairs. Popul Health Metr. 2006;4:2. [PMID: 16606453]

35. Xue JL, St Peter WL, Ebben JP, Everson SE, Collins AJ. Anemia treatment in the pre-ESRD period and associated mortality in elderly patients. Am J Kidney Dis. 2002;40(6): 1153-61. [PMID: 12460033]

DOI:10.1053/ajkd.2002.36861

36. Weisbord SD, Fried LF, Mor MK, Resnick AL, Kimmel PL, Palevsky PM, Fine MJ. Associations of race and ethnicity with anemia management among patients initiating renal replacement therapy. J Natl Med Assoc. 2007;99(11): 1218-26. [PMID: 18020096]

37. Iezzoni LI. Assessing quality using administrative data. Ann Intern Med. 1997;127(8 Pt 2):666-74. [PMID: 9382378]

Submitted for publication August 1, 2009. Accepted in revised form October 14, 2009.

This article and any supplementary material should be cited as follows:

Fischer MJ, Stroupe KT, Hynes DM, Blemur P, Sohn M-W, Browning MM, Huo Z, O’Hare AM, Kaufman JS. Validation of erythropoietin use data on Medicare's End-Stage Renal Disease Medical Evidence Report. J Rehabil Res Dev. 2010;47(8):751-62.

DOI:10.1682/JRRD.2009.08.0108

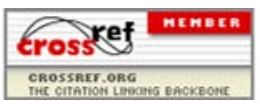

